\title{
On the Use of an Ultra-Tight Integration for Robust Navigation in Jammed Scenarios
}

\section{Cristodaro, Calogero}

INSTITUTE OF NAVIGATION

2019-09-30

Cristodaro , C , Falco , G , Ruotsalainen , L \& Dovis , F 2019, On the Use of an Ultra-Tight Integration for Robust Navigation in Jammed Scenarios . in Proceedings of the 32nd International Technical Meeting of the Satellite Division of The Institute of Navigation (ION GNSS+ 2019) . Institute of Navigation. Satellite Division. Proceedings of the International Technical Meeting , INSTITUTE OF NAVIGATION, Manassas, VA, pp. 2991-3004, International technical meeting of the Satellite Division of the Institute of Navigation, Miami, Florida , United States , 16/09/2019 . https://doi.org/10.33012/2019.16965

http://hdl.handle.net/10138/309988

https://doi.org/10.33012/2019.16965

unspecified

acceptedVersion

Downloaded from Helda, University of Helsinki institutional repository.

This is an electronic reprint of the original article.

This reprint may differ from the original in pagination and typographic detail.

Please cite the original version. 


\title{
On the Use of an Ultra-Tight Integration for Robust Navigation in Jammed Scenarios
}

\author{
Calogero Cristodaro $^{(1)}$, Gianluca Falco ${ }^{(2)}$, Laura Ruotsalainen ${ }^{(3,4)}$, Fabio Dovis ${ }^{(1)}$ \\ (I) Politecnico di Torino, Torino, Italy \\ (2) LINKS Foundation, Torino, Italy \\ (3) University of Helsinki, Helsinki, Finland \\ (4) Finnish Geospatial Research Institute, Helsinki, Finland
}

\section{BIOGRAPHIES}

Calogero Cristodaro received his doctoral degree in Electrical, Electronic and Communications Engineering at Politecnico di Torino, as a member of the Navigation Signal Analysis and Simulation (NavSAS) group. His research is focused on the advanced integration of GNSS and external sensors for autonomous mobility applications. He developed his M.S. thesis at European Space Research and Technology Centre (ESTEC) addressing maritime multipath and ionospheric scintillation effects on GNSS signals. Currently, he is a navigation system engineer at Thales Alenia Space, Italy.

Gianluca Falco received the M.S. degree in communications engineering from Politecnico di Torino, Italy, in 2007 and the Ph.D. degree in electronic and communication in 2011. He is currently a Communication Engineer with the Navigation Technologies Research Area, LINKS Foundation, Turin, Italy. His research interest focuses on multi-sensors fusion, particularly between GPS and inertial navigation systems, and on advanced processing techniques for dual frequency and multi-constellation GNSS receivers.

Laura Ruotsalainen is an Associate Professor of Spatiotemporal Data Analysis for Sustainability Science at the Department of Computer Science at the University of Helsinki, Finland. She is also part-time affiliated at the Finnish Geospatial Research Institute, Finland, where she has worked as e.g. a Research Manager since 2010. She received her doctoral degree in 2013 from the Department of Pervasive Computing, Tampere University of Technology, Finland. Her doctoral research was focused on vision-aided seamless indoor/outdoor pedestrian navigation. Her current research interests cover, in addition to vision-aided navigation, development of sophisticated data analysis means for robust navigation and situational awareness especially in urban areas, for GNSS interference mitigation as well as for utilization of spatiotemporal data for sustainability science.

Fabio Dovis is an associate professor at the Department of Electronics and Telecommunications of Politecnico di Torino as a member of the Navigation Signal Analysis and Simulation (NavSAS) group. His research interests cover the design of GPS and Galileo receivers and advanced signal processing for interference and multipath detection and mitigation. He has a relevant experience in European projects in satellite navigation as well as cooperation with industries and research centers.

\begin{abstract}
The need of accurate and reliable positioning and navigation units is becoming of paramount importance with the advent of new services and new paradigms for transportation systems. Among the multiple technologies and sensors that might be used for positioning, Global Navigation Satellite System (GNSS) can be considered as the core technology and a superior system capable to provide the user with estimates of his own position in a global reference frame. Unfortunately, received GNSS signals are extremely weak and thus vulnerable to non-intentional and intentional Radio Frequency Interference. In particular, the malicious intentional interference produced by jammers is proliferating and it is becoming a serious threat for GNSS receivers. The integration of GNSS and external sensors with complementary characteristics, is the key for overcoming the weaknesses and enhancing the strengths of each sub-system. In this paper, the design of an ultra-tight hybrid navigation system is proposed with the aim to enhance the robustness of the navigation system in presence of jamming. A GNSS unit has been integrated with external sensors, namely Inertial Navigation Systems, visual sensor and odometer. Exploiting the "record and replay" of real GNSS signal artificially augmented with jamming interference within the L1 GNSS bandwidth, the performance has been assessed in controlled scenarios. The results presented in this paper show that the multi-sensor integrated system can sustain the navigation even in presence of severe jamming, although such a strong interference completely masks the GNSS signal denying the navigation of a GNSS stand-alone system.
\end{abstract}


Moreover, the test procedures suitability of the record and replay approach for the jamming scenario generation and combination with real datasets are discussed and analyzed.

\section{INTRODUCTION}

The persistent growth of applications and services based on the user position estimation, together with the advent of new paradigms for transportation systems, is increasing the need of higher accuracy and reliability of the estimated position with respect to what is currently provided by commercial positioning platforms. Modern applications in the road field require different accuracy and continuity of the positioning and, therefore, different requirements are set for the Key Performance Indicator (KPI), depending on the specific application. The more accurate and reliable the positioning information, the more advanced the service it can support. Given this picture, Global Navigation Satellite System (GNSS) can be considered the core technology among the multiple technologies and sensors that might be used for positioning, since it is capable to provide the user with estimates of his own position in a global reference frame. In fact, most of the other systems and positioning technologies provide solutions that are relative to an absolute GNSS position. Unfortunately, received GNSS signals are extremely weak and thus vulnerable to non-intentional and intentional Radio Frequency Interference (RFI). Among these threats, the malicious intentional interference produced by jammers is dangerously proliferating both in number of reported events as well as in relevance, considering the strong chain effect on the applications relying on GNSS. These devices are capable of broadcasting disruptive signals overlapping a large part of the targeted GNSS frequency band thus preventing the operation of GNSS receivers in estimating their position [1]. Despite GNSS jammers are illegal in most countries, they can be easily purchased on-line and their rapid diffusion is becoming a serious threat to satellite navigation.

These aspects raised the concerns for security, since GNSS receivers are expected to operate in more challenging environments with respect to the past, and thus there is the need to develop customized positioning algorithms specifically tailored to the user's requirements. Within this context, one possible strategy to overcome the weaknesses of GNSS, is given by the integration of measurements from multiple sensors with complementary characteristics. The intelligent integration of heterogeneous data not only enhances the performance of the whole positioning system but, at the same time, limits the weaknesses of each individual sensor guaranteeing the user requirements in term of accuracy, cost, coverage, date rate, integrity and more.

Nowadays there is a plethora of sensor technologies available, that can be coupled with GNSS, to reach a robust and reliable navigation solution. Based on the own different nature, each sensor can provide absolute or relative positioning. One of the most used complementary technology is represented by the inertial sensors. An Inertial Navigation System (INS), includes self-contained sensors such as accelerometers and gyroscopes that measure the specific force and the angular rate, respectively [2]. They provide relative position independently from the environment but, unfortunately, they are affected by unbounded errors. Another sensor that can be used for positioning is the camera as a visual sensor. Since cameras are not affected by error sources deteriorating the measurements, which are typical of INS, they might be exploited to track the motion of the features from observed images in order to extract the information about the user motion [3]. Odometers can also retrieve information about the vehicle position by measuring its speed and thus giving information about the traveled distance [4]. In addition, Light Detection and Ranging (LIDAR) [5], Wi-Fi and mobile networks [6], Ultra-Wide Band (UWB) systems [7] are just some other examples of possible integration with GNSS receivers. A survey of the information sources and information fusion technologies used in current in-car navigation systems is presented in [4].

Depending on the level of information exchanged between GNSS and the other components in the integrated system, they can be integrated according to different strategies, commonly referred as loose, tight and ultra-tight (or deep) integration. The terms Loosely Coupled (LC), Tightly Coupled (TC) and Ultra-Tightly Coupled (U-TC) are used hereafter to refer to loose, tight and ultra-tight approach, respectively. These solutions basically differ in the degree of integration of the systems, i.e. for the nature of the information extracted from the systems and used in the hybridization process, as well as for the architecture of the interactions between the systems. LC and TC methods are quite consolidated in the scientific literature. Interested readers can found useful material in [8]-[10]. Similar to vector tracking receiver, U-TC receivers are characterized by the fact that the channels are not tracked individually and independently (i.e. scalar tracking), but they are aided from the navigation filter. By following this approach, once a good Position, Velocity and Time (PVT) solution is available, satellite signals can be continuously tracked even under weak signal conditions. More in details, in case of a GNSS/INS UTC system, the PVT solution is obtained from the GNSS module assisted by the INS and the navigation filter's outputs are also used to compensate the errors affecting the INS and to update the Numerical Control Oscillators (NCO) in each tracked channel of the GNSS module. 
In traditional U-TC GPS/INS systems the mutual assistance between GPS and INS is strengthened so that the signal tracking ability is much improved [12]. However, the performance of a conventional GNSS/INS U-TC system tends to degrade in challenging environments, such as in case of jamming, or high dynamic maneuvers. When low-end Inertial Measurement Units (IMUs) based on MEMS sensors are employed, the performance degradation will be even more severe. To solve this problem, two possible options can be adopted to reinforce the U-TC system: the first one is to use an adaptive Kalman filter (AKF), as done in [13], where the covariance matrixes of process noise (i.e., Q) and measurement noise (i.e., R) of the KF are continuously adapted according to the estimated noise characteristics of the surrounding environment. The second approach does not change the design of the KF but it reinforces the traditional U-TC with measurements coming from additional sensors (e.g. visual camera, odometer, LIDAR, etc.). In this paper, we have adopted the second approach where the GNSS module has been coupled with the following sensors: INS, odometer and a monocular visual camera.

This paper therefore aims at improving the accuracy and availability of a fully autonomous GNSS/INS based navigation solution by adding low-cost sensors such as monocular camera and odometer. The added value of this research, thus, lies in the integration of the aforementioned sensors, performed at the deepest level, as well as in the fact that adding a camera as a vision sensor mitigates the short-term stability of the INS.

Three key contributions are addressed herein the paper: first the proposed camera/odometer/IMU/GNSS integration architecture complements each of the sensors and potentially overcomes the disadvantage of traditional GNSS/IMU integration. Second, although significant effort has been spent on developing visual odometry and attitude algorithms (in particular through a monocular camera), the efficiency and performance regarding image frame rate has yet to be evaluated. Eventually, the integrated system is validated by using a live dataset differently from most of the previous works on the ultra-tight integration, that tested their algorithm only via simulation [11-13].

Indeed, this paper represents a further extension to our previous research works [14] related to the ultra-tight integration of GNSS/INS/vision investigated for jamming mitigation in a static scenario and to [15], where such integration was only preliminary investigated in a dynamic scenario.

This paper is organized as follows. After this introduction, the next section describes the process used to determine the motion and orientation of the receiver by analyzing the associated camera images, followed by the mathematical description of the navigation filter, which is in charge of blending the measurements coming from the aforementioned sensors. The feedback estimation from the navigation filter to the code and carrier NCOs is also explained. Afterwards, the general description of the hardware used in the data collection is presented, with emphasis to the artificial generation of jamming. The performance results are then summarized for different power level of the jamming signals and, eventually, some conclusions are drawn.

\section{PROPOSED ULTRA-TIGHT INTEGRATION SCHEME}

The first part of the section provides a description of the measurements coming from a camera while the descriptions of measurements coming from INS [2] and odometer [4] are omitted since they are considered well known and state of art. Then, the second part of the section deals with the mathematical aspects concerning the design of the navigation filter (according to the proposed U-TC integration) that makes the hybridized receiver more robust to jamming attacks.

\subsection{Monocular Camera as a Visual Sensor}

Imaging sensors are widely used for navigation due to the possibility to extract not only spatial information, but also geometric relations between the camera and the real world. The estimation of the ego-motion of a body using the information contained in the images acquired by a camera, known as Visual Odometry (VO), incrementally estimates the camera pose by observing the changes that motion induces on the images, i.e. correspondent features. This motion provides relative positioning information, i.e. speed and attitude of the user [3], and it is represented by a three-dimensional rigid transformation between subsequent frames, which is composed by rotation and translation.

This relation is equivalent up to a scale factor, which means solving the distance between the camera center and the 3D object scene. Usually, this scale factor is computed by using two cameras (stereo-camera), provided that their baseline is known [18]. However, the distance between the two cameras affects the accuracy of the motion estimation obtained from images. The farther the two cameras are from each other the better the accuracy is. Although monocular camera suffers from a phenomenon called scale ambiguity (due to the unknown depth of an object), it might also be exploited to retrieve information about the distance of the objects being photographed. 
In our implementation, the visual sensor is a monocular camera due to its low prize and small size. The scale ambiguity problem is solved by using the concepts of visual gyroscope and visual odometer, presented in [19].

\section{Visual Gyroscope}

The visual gyroscope monitors the attitude of the camera by tracking the motion of virtual features in consecutive images called vanishing points, which can be considered as the image point where the lines parallel in the scene appear to intersect. A graphical representation is reported in Figure 1 as an example, where the vanishing point is estimated within an urban environment.

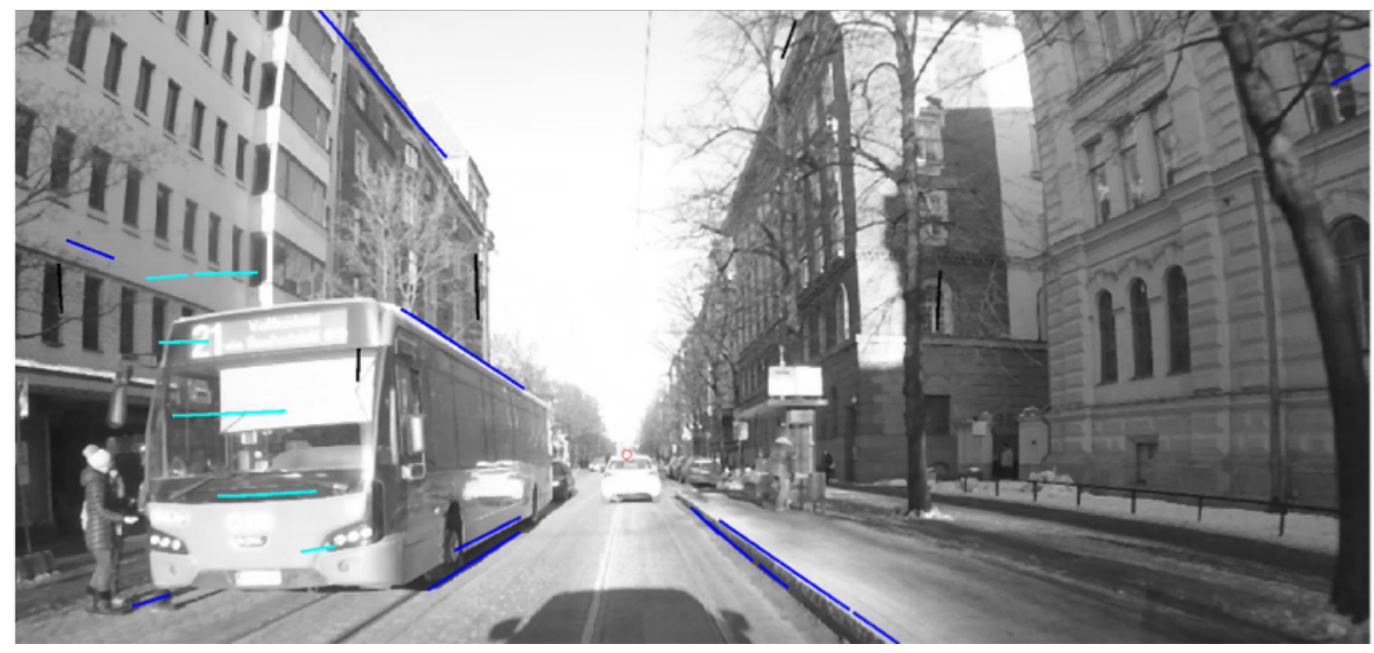

Figure 1: Example of vanishing points representation, determined in urban environment. Horizontal (turquoise) and along the direction of propagation (blue) lines. Central vanishing point (red dot).

The vanishing point in the propagation direction (z-axis) is called the central vanishing point. The three vanishing point locations are defined by the camera orientation with respect to the scene and the camera intrinsic parameters. This relation is described with

$$
\mathbf{V}=\mathbf{K R}
$$

where $\mathbf{V}$ is the vanishing point location matrix $\left[\mathbf{v}_{x}, \mathbf{v}_{y}, \mathbf{v}_{z}\right], \mathbf{R}$ is the camera rotation matrix and $\mathbf{K}$ is the calibration matrix containing the camera intrinsic parameters, i.e. the focal length $\left(f_{x}, f_{y}\right)$, the principal point $(u, v)$, and skew coefficient $S$. The central vanishing point $\mathbf{v}_{z}$, presented in homogenous coordinates as $(x, y, 1)$, where the $x$ and $y$ are the pixel coordinate, can be written as

$$
\mathbf{v}_{z}=\left[\begin{array}{c}
f_{x} \sin \theta+u \cos \phi \cos \theta \\
-f_{y} \sin \phi \cos \theta+v \cos \phi \cos \theta \\
\cos \phi \cos \theta
\end{array}\right]
$$

Therefore, the heading change $(\theta)$ and pitch $(\phi)$ can be finally obtained as

$$
\begin{gathered}
\theta=\arcsin \left(\frac{x-u}{f_{x}}\right) \\
\phi=\arcsin \left(\frac{y-v}{-f_{y} \cos \theta}\right)
\end{gathered}
$$

The logic behind the selection of the central vanishing point is a voting scheme. Basically, any vanishing point candidate is voted for all the intersection points of lines found. Eventually, the one getting most of the votes is selected as the correct one. A detailed explanation can be found in [19]. Moreover, in order to evaluate the accuracy of the estimated vanishing point, the concept of Line Dilution Of Precision (LDOP) is used. It basically consists of a dilution of precision value demonstrating the geometry of the lines used for calculating the position of the vanishing point. A large LDOP value, leads to large uncertainty of the vanishing point location which can be weighted accordingly into the navigation filter. This concept is explained in detail in [19].

\section{$\underline{\text { Visual Odometer }}$}


The visual odometer measures the user translation between consecutive images. The relation between two points found from a plane in consecutive images, from an image point $\widehat{\mathbf{x}}$ to an image point $\hat{\mathbf{x}}^{\prime}$, is called homography and can be written as [3]

$$
\hat{\mathbf{x}}^{\prime}=\mathbf{R} \hat{\mathbf{x}}+\mathbf{t} / \mathrm{Z}
$$

where $\mathbf{K}$ is the camera calibration matrix, $\mathbf{R}$ is camera rotation matrix, $\mathbf{t}$ is the translation of the camera origin and $\mathrm{Z}$ is the depth of the point.

In our implementation, the distance $\mathrm{Z}$ from the camera to the object is resolved by using a special configuration of the camera. In this context, the camera is facing the road with an attitude resolved with visual gyroscope and known height of the camera. In particular, the object has coordinates $(\mathrm{X}, \mathrm{Y}, \mathrm{Z})$ and it is projected into image point $(\mathrm{x}, \mathrm{y}, \mathrm{z}) . \phi$ is the pitch of the camera whereas $\beta$ is the angle between the principal ray of the camera and the ray from the camera to the object using the image point $y$ and the focal length $f_{y}$. $h$ is the height of the camera with respect to the ground. Following the mathematical derivation in [19], the depth $\mathrm{Z}$ of the object is estimated as

$$
\mathrm{Z}=\frac{h \cos \beta}{\sin (\phi+\beta)}
$$

$\mathrm{Z}$ can be finally used to find the final expression of the translation $\mathbf{t}$.

\subsection{Navigation Filter Design}

The block diagram of the whole system is shown in Figure 2. The central navigation filter is an error state EKF that accepts the GNSS inputs from the signal tracking channels, which is able to process both GPS and Galileo signals. The other inputs are given by the other aforementioned sensors: INS, visual sensor and odometer. On the other hand, the central navigation filter outputs the corrections for the INS and the predicted pseudoranges and pseudoranges-rate in order to update the NCOs.
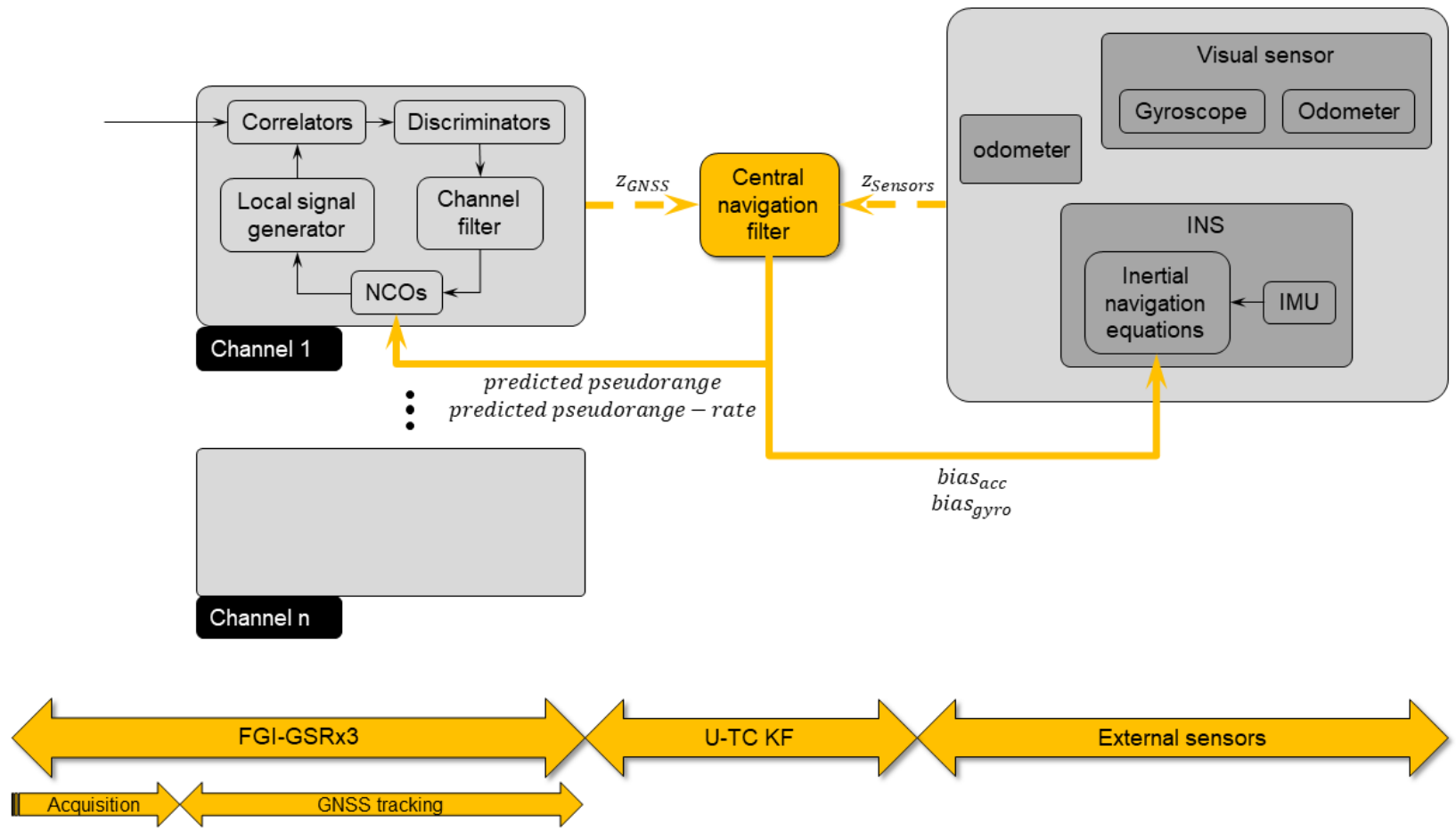

Figure 2: Block diagram of the proposed multi-sensor U-TC GNSS receiver. 
The details of the navigation filter, implemented according to an EKF scheme, used within the architecture in Figure 2, are presented in the following.

\section{State-space model}

The state-space model, expressed in the discrete-time domain, can be written as

$$
\Delta \mathbf{x}[\mathrm{n}]=\left[\Delta \mathbf{p}^{e}[\mathrm{n}]^{T}, \Delta \mathbf{v}^{e}[\mathrm{n}]^{T}, \Delta \boldsymbol{\psi}^{e}[\mathrm{n}]^{T}, \mathbf{b}_{\mathbf{a}}{ }^{\mathrm{b}}[\mathrm{n}]^{T}, \mathbf{b}_{\mathbf{g}}{ }^{\mathrm{b}}[\mathrm{n}]^{T}, \quad \Delta \boldsymbol{\tau}[\mathrm{n}], \Delta v_{\tau}[\mathrm{n}]\right]^{T} \in R^{1 \&, 11}
$$

where the superscripts ${ }^{e, b}$ indicates ECEF frame and body frame, respectively. The state vector stores the following components:

- $\Delta \mathbf{p}^{\mathbf{e}}[\mathrm{n}] \in R^{3,1}$ is the corrections vector to be applied to the nominal body position at the time instant $n$, expressed in the ECEF frame;

- $\quad \Delta \mathbf{v}^{\mathrm{e}}[\mathrm{n}] \in \mathrm{R}^{3,1}$ is the corrections vector to be applied to the nominal body velocity at the time instant $n$, expressed in the ECEF frame;

- $\quad \Delta \boldsymbol{\Psi}^{\mathrm{e}}[\mathrm{n}] \in \mathrm{R}^{3,1}$ is the vector of misalignment angles along each axis (attitude corrections) at the time instant $n$, expressed in the ECEF frame;

- $\quad \mathbf{b}_{\mathrm{a}}{ }^{\mathrm{b}}[\mathrm{n}] \in \mathrm{R}^{3, \mathbb{1}}$ is the vector of the biases of the accelerometers at the time instant $n$, expressed in the body frame;

- $\quad \mathbf{b}_{\mathrm{g}}{ }^{\mathrm{b}}[\mathrm{n}] \in \mathrm{R}^{3,1}$ is the vector of the biases of the gyroscopes at the time instant $n$, expressed in the body frame;

- $\Delta \boldsymbol{\tau}[\mathrm{n}] \in R^{2,1}$ is the receiver clock bias for GPS and Galileo expressed in meters;

- $\quad \Delta \mathrm{v}_{\tau}[\mathrm{n}] \in \mathrm{R}^{\mathbb{1}, \mathbb{1}}$ is the receiver clock drift expressed in meters per second.

\section{$\underline{\text { State transition matrix }}$}

The state transition matrix $\boldsymbol{\Phi}[n]$ of the discrete-time state-space model can be defined as:

$$
\boldsymbol{\Phi}[n]=\left[\begin{array}{ccccccc}
\mathbf{I}_{3} & \mathrm{~T}_{\mathrm{c}} \mathbf{I}_{3} & \mathbf{0}_{3} & \mathbf{0}_{3} & \mathbf{0}_{3} & \mathbf{0}_{3 \times 2} & \mathbf{0}_{3 \times 1} \\
\mathbf{N}^{\mathrm{e}} & \mathbf{I}_{3}-2 \mathrm{~T}_{\mathrm{c}} \boldsymbol{\Omega}_{\mathrm{ie}}^{\mathrm{e}} & -\mathrm{T}_{\mathrm{c}} \mathbf{F}^{\mathbf{e}}[\boldsymbol{n}] & \mathrm{T}_{\mathrm{c}} \mathbf{C}_{\mathrm{b}}^{\mathrm{e}}[\mathrm{n}] & \mathbf{0}_{3} & \mathbf{0}_{3 \times 2} & \mathbf{0}_{3 \times 1} \\
\mathbf{0}_{3} & \mathbf{0}_{3} & \mathbf{I}_{3}-\mathrm{T}_{\mathrm{c}} \boldsymbol{\Omega}_{\mathrm{ie}}^{\mathrm{e}} & \mathbf{0}_{3} & -\mathrm{T}_{\mathrm{c}} \mathbf{C}_{\mathrm{b}}^{\mathrm{e}}[\mathrm{n}] & \mathbf{0}_{3 \times 2} & \mathbf{0}_{3 \times 1} \\
\mathbf{0}_{3} & \mathbf{0}_{3} & \mathbf{0}_{3} & \mathbf{I}_{3}+\mathrm{T}_{\mathrm{c}} \mathbf{D}_{\mathrm{a}} & \mathbf{0}_{3} & \mathbf{0}_{3 \times 2} & \mathbf{0}_{3 \times 1} \\
\mathbf{0}_{3} & \mathbf{0}_{3} & \mathbf{0}_{3} & \mathbf{0}_{3} & \mathbf{I}_{3}+\mathrm{T}_{\mathrm{c}} \mathbf{D}_{\mathrm{g}} & \mathbf{0}_{3 \times 2} & \mathbf{0}_{3 \times 1} \\
\mathbf{0}_{2 \times 3} & \mathbf{0}_{2 \times 3} & \mathbf{0}_{2 \times 3} & \mathbf{0}_{2 \times 3} & \mathbf{0}_{2 \times 3} & \mathbf{I}_{2} & \mathbf{T}_{\mathrm{c}_{2 \times 1}} \\
\mathbf{0}_{1 \times 3} & \mathbf{0}_{1 \times 3} & \mathbf{0}_{1 \times 3} & \mathbf{0}_{1 \times 3} & \mathbf{0}_{1 \times 3} & \mathbf{0}_{1 \times 2} & 1
\end{array}\right] \in \mathrm{R}^{1 \mathbb{1}, 1 \mathbb{R}}
$$

where $T_{c}$ is the sampling interval, $\boldsymbol{N}^{e}$ is the tensor of gravity gradients, $\boldsymbol{\Omega}_{\mathrm{ie}}^{e}$ is the Earth rotation rate, $\mathbf{F}^{e}$ is the skew symmetric matrix of the accelerometers measurements, $\mathbf{C}_{\mathrm{b}}^{\mathrm{e}}$ is the rotation matrix from the body frame to the Earth frame, $\mathbf{D}$ is the time-constant diagonal matrix that defines a first-state Gauss-Markov model.

The discrete-time state-space model is written as:

$$
\Delta \mathbf{x}[\mathrm{n}+1]=\boldsymbol{\Phi}[\mathrm{n}] \Delta \mathbf{x}[\mathrm{n}]+\boldsymbol{\Gamma}[\mathrm{n}] \boldsymbol{\eta}[\mathrm{n}]
$$

with the definition of the model noise vector as $\mathbf{w}[n]=\boldsymbol{\Gamma}[n] \boldsymbol{\eta}[n]$

$$
\begin{aligned}
& \boldsymbol{\eta}[n]=\left[\boldsymbol{\eta}_{a}[n]^{\mathrm{T}}, \boldsymbol{\eta}_{\mathbf{g}}[n]^{\mathrm{T}}, \boldsymbol{\eta}_{\mathrm{aa}}[n]^{\mathrm{T}}, \boldsymbol{\eta}_{\mathrm{gg}}[\mathrm{n}]^{\mathrm{T}}, \boldsymbol{\tau}[n]^{\mathrm{T}}, v_{\tau}[n]\right]^{\mathrm{T}} \in \mathrm{R}^{15,1}
\end{aligned}
$$

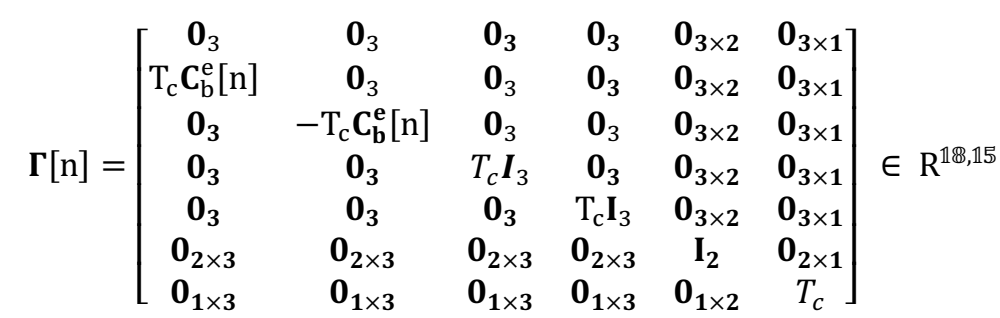


where $\boldsymbol{\eta}_{\boldsymbol{a}}$ is a driving noise term acting on the accelerometers in the body frame, $\boldsymbol{\eta}_{\boldsymbol{g}}$ is a driving noise term acting on the gyroscopes in the body frame, $\boldsymbol{\eta}_{\boldsymbol{a} \boldsymbol{a}}$ is the driving noise for the accelerometers biases, $\boldsymbol{\eta}_{\boldsymbol{g} \boldsymbol{g}}$ is the driving noise for the gyroscopes biases.

\section{Measurements equation}

The observation vector has been augmented with the measurements coming from visual sensor and odometer (i.e. $\mathbf{z}_{\text {ext }}[n]$ ), and can be written as

$$
\Delta \mathbf{z}[\mathrm{n}]=\left[\begin{array}{c}
\Delta \mathbf{z}_{\mathrm{GNSS}}[\mathrm{n}] \\
\Delta \mathbf{z}_{\mathrm{ext}}[\mathrm{n}]
\end{array}\right] \in \mathrm{R}^{2 \mathrm{~N}_{\mathrm{sat}}+\Phi, \mathbb{1}}
$$

$\Delta \mathbf{z}_{G N S S}[n]$ includes both (i) the pseudorange residuals (i.e. $\tilde{\boldsymbol{\varepsilon}}_{\text {code }}[n]$ produced by the code discriminators), and (ii) the difference between the GNSS measured pseudorange rates $\dot{\mathbf{r}}[n]$ and the estimated ones $(\breve{\mathbf{r}}[n])$ computed through the INS measurements. Thus, in formulae, $\Delta \mathbf{z}_{G N S S}[n]$ can be written as

$$
\Delta \mathbf{z}_{\mathrm{GNSS}}[\mathrm{n}]=\left[\tilde{\boldsymbol{\varepsilon}}_{\text {code }}[\mathrm{n}]^{\mathrm{T}},\left[\dot{\mathbf{r}}[\mathrm{n}]^{\mathrm{T}}-\breve{\mathbf{r}}[\mathrm{n}]^{\mathrm{T}}\right]\right]^{\mathrm{T}} \in \mathrm{R}^{2 N_{\text {sat }}, \mathbb{1}}
$$

$\Delta \mathbf{z}_{\text {ext }}[\mathrm{n}]$ is defined as

$$
\Delta \mathbf{z}_{\text {ext }}[\mathrm{n}]=\overline{\boldsymbol{\Theta}}[\mathrm{n}]-\boldsymbol{\Theta}_{\mathrm{INS}}[\mathrm{n}] \in \mathrm{R}^{\Phi, \mathbb{1}}
$$

where the vectors $\overline{\boldsymbol{\Theta}}[\mathrm{n}]$ and $\boldsymbol{\Theta}_{\mathrm{INS}}[n]$ are stated as in (9)-(10) and expressed in the ECEF frame

$$
\begin{gathered}
\overline{\boldsymbol{\Theta}}[\mathrm{n}]=\left[\overline{\boldsymbol{\psi}}_{\text {vis }}^{e}[n]^{T}, \overline{\mathbf{v}}_{v i s}^{e}[n]^{T}, \overline{\mathbf{v}}_{\text {odo }}^{\mathrm{e}}[n]^{T}\right]^{\mathrm{T}} \in \mathrm{R}^{\Phi, \mathbb{1}} \\
\boldsymbol{\Theta}_{\mathrm{INS}}[\mathrm{n}]=\left[\boldsymbol{\psi}_{\text {INS }}^{e}[\mathrm{n}]^{\mathrm{T}}, \mathbf{v}_{I N S}^{e}[\mathrm{n}]^{\mathrm{T}}, \mathbf{v}_{I N S}^{e}[\mathrm{n}]^{\mathrm{T}}\right]^{\mathrm{T}} \in \mathrm{R}^{\Phi, \mathbb{1}}
\end{gathered}
$$

where

- $\overline{\boldsymbol{\psi}}_{v i s}^{e}[\mathrm{n}]$ is the vector of attitude Euler angles estimated by the visual gyroscope;

- $\quad \overline{\mathbf{v}}_{v i s}^{e}[\mathrm{n}]$ is the vector of velocity estimated by the visual odometer;

- $\overline{\mathbf{v}}_{\mathrm{odo}}^{\mathrm{e}}[\mathrm{n}]$ is the vector velocity estimated by the odometer;

- $\boldsymbol{\psi}_{I N S}^{e}[\mathrm{n}]$ is the vector of attitude Euler angles estimated by the INS, as described in [2];

- $\mathbf{v}_{I N S}^{e}[\mathrm{n}]$ is the vector of velocity estimated by the INS, as in [2].

\section{$\underline{\text { H matrix definition }}$}

The matrix $\mathbf{H}[\mathrm{n}]$, that relates the measurements vector with the states, has been augmented with the measurements coming from visual sensor and odometer $\left(\mathbf{H}_{\mathrm{ext}}[\mathrm{n}]\right)$, and can be written as

$$
\mathbf{H}[\mathrm{n}]=\left[\begin{array}{c}
\mathbf{H}_{\mathrm{GNSS}}[\mathrm{n}] \\
\mathbf{H}_{\mathrm{ext}}[\mathrm{n}]
\end{array}\right] \in \mathrm{R}^{2 \mathrm{~N}_{\mathrm{sat}}+\Phi, 1 \mathbb{B}}
$$

where the component $\mathbf{H}_{\mathrm{GNSS}}[\mathrm{n}]$ can be written as in (12)

$$
\mathbf{H}_{G N S S}[\mathrm{n}]=\left[\begin{array}{ccccccc}
-\mathbf{u}_{\mathrm{e}}[\mathrm{n}] & \mathbf{0}_{\mathrm{N}_{\mathrm{sat}} \times 3} & \mathbf{0}_{\mathrm{N}_{\mathrm{sat}} \times 3} & \mathbf{0}_{\mathrm{N}_{\mathrm{sat}} \times 3} & \mathbf{0}_{\mathrm{N}_{\mathrm{sat}} \times 3} & \mathbf{1}_{\mathrm{N}_{\mathrm{sat}} \times 2} & \mathbf{0}_{\mathrm{N}_{\mathrm{sat}} \times 1} \\
\mathbf{0}_{\mathrm{N}_{\mathrm{sat}} \times 3} & -\mathbf{u}_{\mathrm{e}}[\mathrm{n}] & \mathbf{0}_{\mathrm{N}_{\mathrm{sat}} \times 3} & \mathbf{0}_{\mathrm{N}_{\mathrm{sat}} \times 3} & \mathbf{0}_{\mathrm{N}_{\mathrm{sat}} \times 3} & \mathbf{0}_{\mathrm{N}_{\mathrm{sat}} \times 2} & \mathbf{0}_{\mathrm{N}_{\mathrm{sat}} \times 1}
\end{array}\right] \in R^{2 N_{s a t}, 1 \mathbb{B}}
$$

where $\mathbf{u}_{\mathrm{e}}[\mathrm{n}]$ is the line-of-sight vector from the receiver to the satellite.

The additional component $\mathbf{H}_{\text {ext }}[\mathrm{n}]$ is related to the additional sensors and it can be expressed as 


$$
\mathbf{H}_{\text {ext }}[\mathrm{n}]=\left[\begin{array}{c}
\mathbf{H}_{\mathrm{vis}}[\mathrm{n}] \\
\mathbf{H}_{\text {odo }}[\mathrm{n}]
\end{array}\right] \in \mathrm{R}^{\Phi, 118}
$$

where

1. $\mathbf{H}_{\mathrm{vis}}[\mathrm{n}]$ can be written as

$$
\mathbf{H}_{\mathrm{vis}}[\mathrm{n}]=\left[\begin{array}{ccccccc}
\mathbf{0}_{3} & \mathbf{0}_{3} & \mathbf{I}_{3} & \mathbf{0}_{3} & \mathbf{0}_{3} & \mathbf{0}_{3 \times 2} & \mathbf{0}_{3 \times 1} \\
\mathbf{D}_{\text {pos }}[\mathrm{n}] & \mathbf{I}_{\mathbf{3}} & \mathbf{0}_{\mathbf{3}} & \mathbf{0}_{3} & \mathbf{0}_{3} & \mathbf{0}_{3 \times 2} & \mathbf{0}_{3 \times 1}
\end{array}\right] \in \mathrm{R}^{6,118}
$$

and $\mathbf{D}_{\text {pos }}[\mathrm{n}]$, according to [14] is equal to

$$
\mathbf{D}_{\text {pos }}[n]=\left[\begin{array}{ccc}
\Delta v_{x}[n] \cos (\theta[n]) T c & 0 & 0 \\
0 & \Delta v_{y}[n] \sin (\theta[n]) T c & 0 \\
0 & 0 & \Delta v_{z}[n] T c
\end{array}\right] \in R^{3,3}
$$

where $\theta[\mathrm{n}]$ is the yaw estimated by the INS; $\Delta v_{x, y, z}$, are the difference between the velocity estimated by the IMU and the visual odometer, expressed in the ECEF frame.

2. $\mathbf{H}_{\text {odo }}[\mathrm{n}]$ can be written as

$$
\mathbf{H}_{\text {odo }}[\mathrm{n}]=\left[\begin{array}{lllllll}
\mathbf{0}_{3} & -\mathbf{I}_{3} & -\grave{\mathbf{v}}_{I N S}^{e}[\mathrm{n}] & \mathbf{0}_{3} & \mathbf{0}_{3} & \mathbf{0}_{3 \times 2} & \mathbf{0}_{3 \times \mathbf{1}}
\end{array}\right] \in \mathrm{R}^{3,1 \mathbb{1}}
$$

where $\grave{\mathbf{v}}_{I N S}^{e}[n]$ is the skew matrix of the velocity estimated by the IMU, expressed in the ECEF frame.

\subsection{NCOs update}

The feedbacks from the central navigation filter to the NCOs are computed from the prediction of the code rate and Doppler frequency which is derived from the inertial system corrected through the bias estimates of the EKF, as shown by the block diagram in Figure 2. The code frequency can be computed in the discrete domain as [17]:

$$
\hat{\mathbf{f}}_{\text {code }}[n]=\left[f_{\text {code }}-\frac{f_{\text {code }}}{c} \frac{\Delta \widehat{\boldsymbol{\rho}}[n]}{T_{c}}\right]^{T} \in R^{N_{s a t}, \mathbb{1}}
$$

where $f_{\text {code }}$ is the nominal code frequency, expressed in $\mathrm{Hz} ; c$ is the speed of light, expressed in $\mathrm{m} / \mathrm{s} ; T_{c}$ is the sampling interval, expressed in $\mathrm{s} ; \Delta \widehat{\boldsymbol{\rho}}[n]=\widehat{\boldsymbol{\rho}}[n]-\widehat{\boldsymbol{\rho}}[n-1]$ is vector of difference between the pseudoranges, estimated by the central navigation filter at instant $n$ and $n-1$, expressed in meters.

On the other hand, the carrier frequency can be computed as [17]:

$$
\hat{\mathbf{f}}_{c a r r}[n]=\left[f_{I F}-\frac{f_{c a r r}}{c} \frac{\hat{\boldsymbol{r}}[n]}{T_{c}}+\frac{f_{\text {carr }}}{c} \Delta v_{\tau}[n]\right]^{T} \in R^{N_{s a t}, \mathbb{1}}
$$

where $f_{I F}$ is the intermediate frequency of the signal, expressed in $\mathrm{Hz} ; f_{\text {carr }}$ is the carrier frequency of the signal, expressed in $\mathrm{Hz}$; $\hat{\hat{r}}[n]$ is the vector of the pseudorange rates, estimated by the central navigation filter at instant $n$, expressed in $\mathrm{m} / \mathrm{s} ; \Delta v_{\tau}[n]$ is the receiver clock drift, expresses in meters per second.

\section{PERFORMANCE ASSESSMENT IN A JAMMED SCENARIO}

This Section includes the performance assessment of the multi-sensor hybridized receiver, done in scenarios characterized by the presence of jamming interference. Moreover, the performance of U-TC navigation solution has been compared with the one obtained 
by considering $\mathrm{TC}$ architectures of the hybridized receiver, in order to evaluate the real improvements of U-TC with respect to TC under weak GNSS signal conditions.

\subsection{Test Scenario Description and HW Setup}

The scenario used for the performance assessment is characterized by good visibility of the GNSS signals since they are not blocked by high buildings and they are not affected by severe multipath. The data collection was performed on May 19, 2017 on a parking within the campus of Politecnico di Torino, Italy. A snapshot of the described environment is illustrated in Figure 3 (left).

The system setup used for recording the data is shown in Figure 3 (right) showing the kart where the sensors are mounted. The GNSS recording system consists of a USRP B210 [22], synchronized to a Rubidium frequency standard, and used to record the digital samples of the GNSS signal. It is configured by using the parameters listed in Table 1. GNSS navigation data are logged by a consumer-grade receiver [23] and its velocity components eventually exploited for emulating the odometer. A consumer-grade MEMS IMU XSens MTi-G-700 [24] logs the specific forces and the angular measurements. The camera used for visual sensor measurements is a GoPro Hero5 Session [25]. The power supply for the recording system is provided by an external battery.
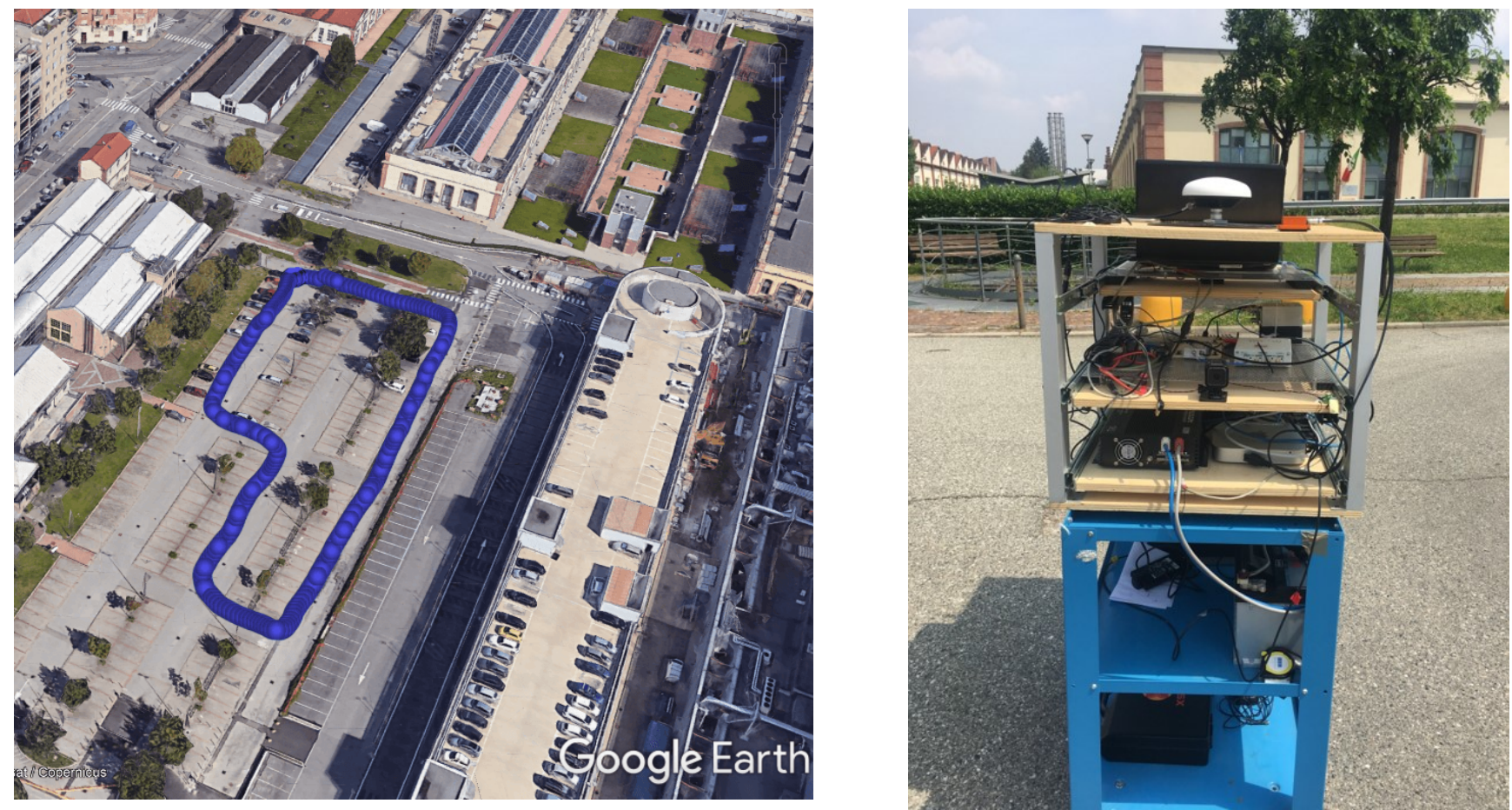

Figure 3: 2D trajectory, Torino, Italy, Image from Google Earth (left). Kart used during the data collection (right).

Table 1: USRP B210 configuration parameters.

\begin{tabular}{|c|c|}
\hline $\begin{array}{c}\text { Configuration } \\
\text { parameter }\end{array}$ & Value \\
\hline$f_{I F}$ & $0 \mathrm{~Hz}$ (baseband) \\
\hline$f_{s}$ & $10 \mathrm{MHz}$ \\
\hline Sampling Type & I and Q \\
\hline
\end{tabular}




\begin{tabular}{|c|c|}
\hline Quantization & 16 bits \\
\hline Interface & USB \\
\hline Reference & Rubidium \\
\hline
\end{tabular}

\subsection{Generation of Jammed Scenarios}

The Record and Replay approach has been exploited in order to create realistic jammed scenarios. Such a technique relies on the reconstruction of the RF signal from the recorded scenario at Intermediate Frequency (IF). As a result, it allows to create a synthetic but realistic scenario in a controlled environment. This methodology puts together the benefits of having a realistic scenario as a baseline and the possibility to have under control the interference power level. Moreover, it enables the possibility of capturing a specific event from the real world and then replay as many times as desired under exactly the same conditions, for deeper and refined analyses. Interested readers can find more details on [20-21] where the topic is discussed thoroughly supported by the presentation of experimental results.

The setup used to inject the jamming signals into the pre-recorded GNSS data, is depicted in Figure 4. The USRP B210 [22] is used to record the digital samples of the GNSS signal as well as to playback them set according to the parameters listed in Table 1. The signal $y_{I F}[n]$ is up-converted back to RF, obtaining the replica $\hat{y}_{R F}(t)$, thanks to the choice of the parameters that grants the fidelity of the scenario during the recording phase, and then combined with the interfering jamming signal $i(t)$. As a result, the interfered signal $\overline{\bar{y}}_{R F}(t)$, is fed to the recording system, creating the digital version $\overline{\bar{y}}_{I F}[n]$ and stored on a memory.

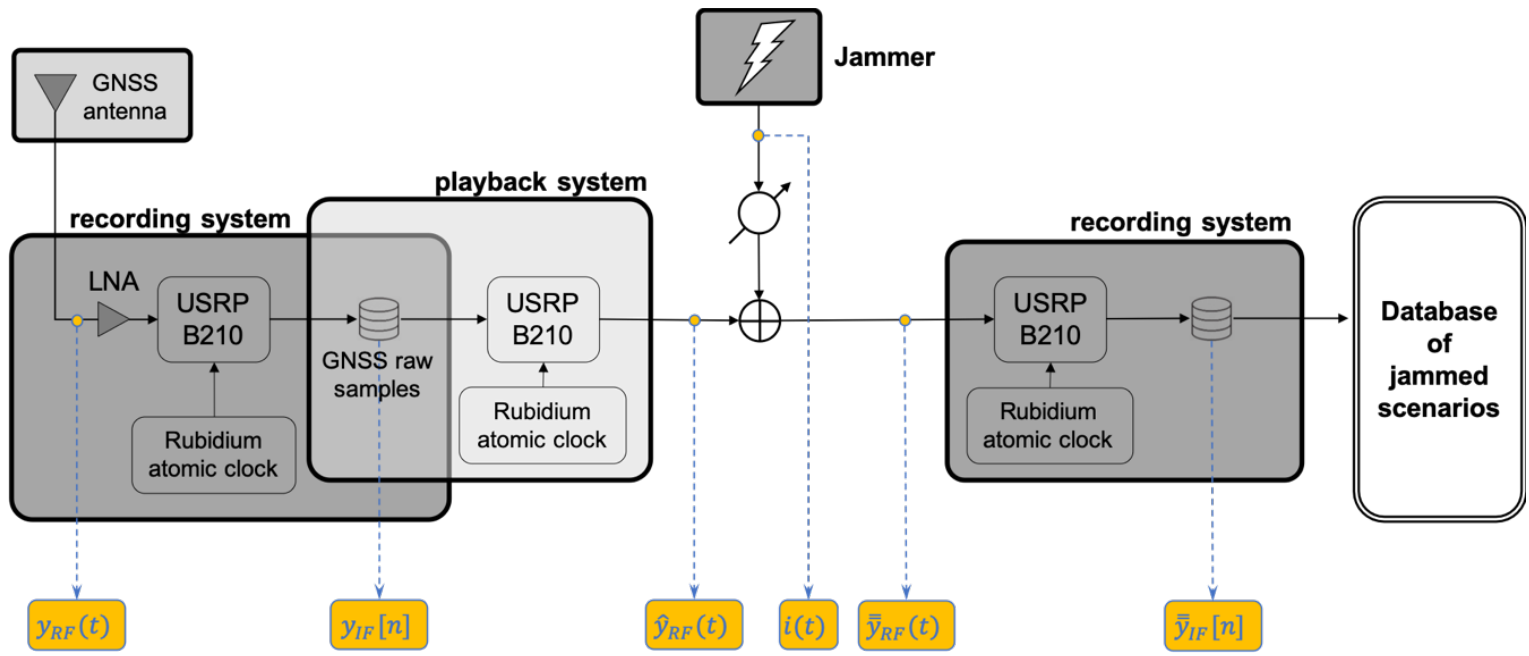

Figure 4: Record and Replay used for the generation of the interfered signal $\overline{\bar{y}}_{R F}(t)$ obtained mixing $i(t)$ and $\hat{y}_{R F}(t)$. Block diagram.

In order to emulate jamming signals with different signal strengths, the power emitted by the jammer is controlled using a variable hardware attenuator. Three different scenario datasets are created, each characterized by different level of attenuation equal to -30 $\mathrm{dB},-20 \mathrm{~dB}$ and $-10 \mathrm{~dB}$, and denoted respectively as jamLight, jamMedium and jamStrong. The power spectral density of the received signals, for all the experiments, is shown in Figure 5 (left). The $\mathrm{C} / \mathrm{N} 0$ as estimated by the GNSS software receiver, considering GPS PRN31, is shown in Figure 5 (right). In the interference-free portion of the signal, the four estimated perfectly overlap, and this is an implicit proof that the replay and new recording phase have not significantly altered the information of the originally recorded signal. Once the interference is injected, it is possible to see the impact of the interfering signal on the measured C/N0. Considering the experiment in which the GNSS signal is affected by jamming signal with the strongest power (purple line), it is possible to observe a drop of the $\mathrm{C} / \mathrm{N} 0$ of about $20 \mathrm{~dB}-\mathrm{Hz}$. 

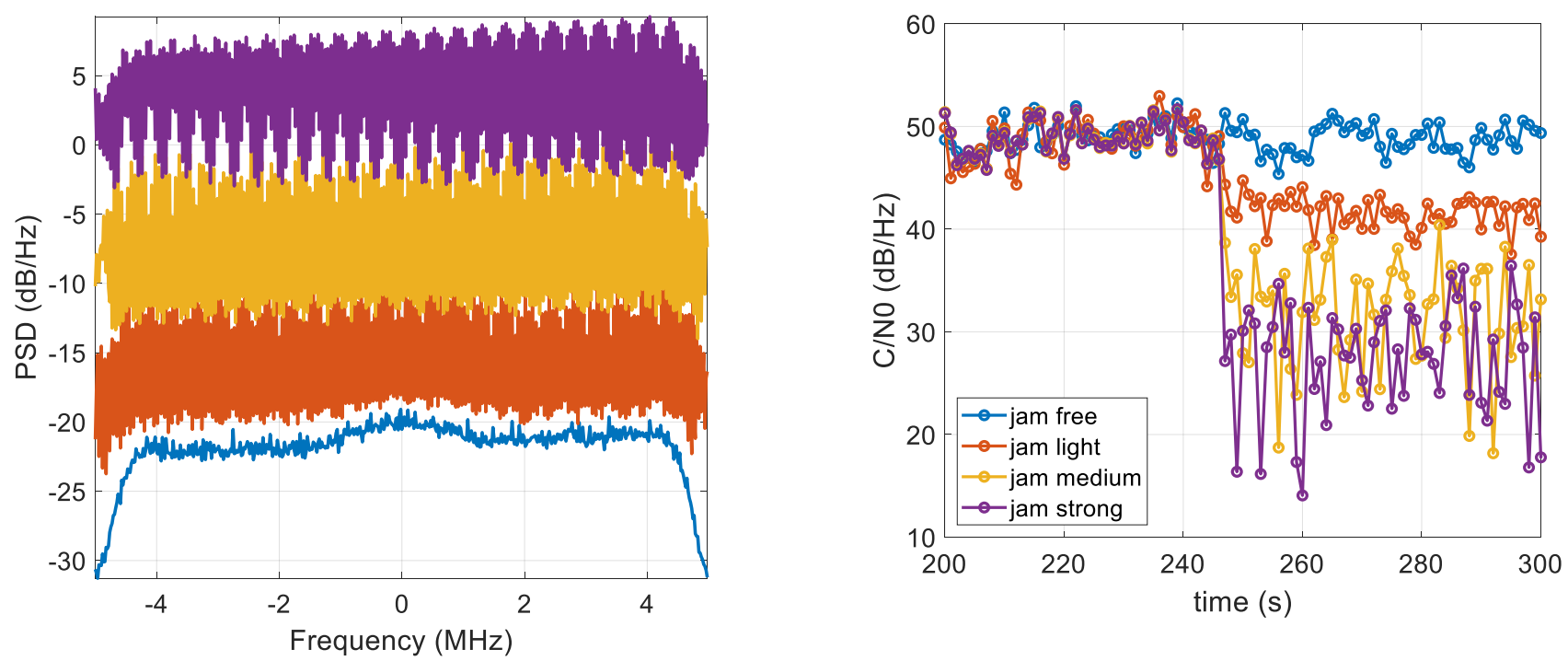

Figure 5: Estimation of PSD (left) and C/NO (right) for different jamming power signal strengths.

\subsection{Positioning Results}

The assessment of the performance evaluation of the U-TC multi-sensor integration (i.e. INS, camera and odometer) is carried out by considering the Horizontal Positioning Accuracy (HPE). The position solutions are reported in Figure 6 and Figure 7 showing the 2D trajectory and the HPE plotted over time, respectively. The statistical assessments of the position solution accuracy together with the availability are summarized in Table 2 .

Table 2: Positioning performance assessment in case of multi-sensor fusion

\begin{tabular}{|c|c|c|c|c|c|c|c|c|}
\hline & \multicolumn{2}{|c|}{ Jam free } & \multicolumn{2}{c|}{ Jam light } & \multicolumn{2}{c|}{ Jam medium } & \multicolumn{2}{c|}{ Jam strong } \\
\hline & TC & U-TC & TC & U-TC & TC & U-TC & TC & U-TC \\
\hline mean(HPE) (m) & 2.09 & 3.40 & 7.05 & 3.32 & - & 3.98 & - & 6.78 \\
\hline $\max (\mathrm{HPE})(\mathrm{m})$ & 4.45 & 4.62 & 14.85 & 4.17 & - & 5.04 & - & 18.04 \\
\hline $50^{\text {th }}$ percentile of HPE (m) & 2.21 & 3.51 & 9.39 & 3.30 & - & 3.97 & - & 4.08 \\
\hline $75^{\text {th }}$ percentile of HPE (m) & 3.00 & 3.91 & 11.65 & 3.57 & - & 4.17 & - & 10.42 \\
\hline $95^{\text {th }}$ percentile of HPE (m) & 3.88 & 4.28 & 13.76 & 3.85 & - & 4.55 & - & 17.19 \\
\hline Availability (\%) & 100 & 100 & 100 & 100 & 0 & 100 & 0 & 100 \\
\hline
\end{tabular}

By analyzing the statistical HPE in Table 2, the first remark is that U-TC integration is the only strategy capable to give a position solution in strongly degraded GNSS signal environments for the $100 \%$ of the simulation time, even if with different accuracy performance along the track. This result can be deduced by observing the HPE of U-TC jamStrong (black curve) in Figure 7: it shows a value of about $0.5 \mathrm{~m}$ in the portion of the trajectory that is interference-free; then it starts increasing once the jamming is injected, with the same rate as jamLight and jamMedium and for about $130 \mathrm{~s}$. Eventually, it reaches the maximum value of HPE (i.e. equal to $18.04 \mathrm{~m}$ ) at the end of the trajectory but no loss of lock in the tracking stage is detected. On the contrary, a TC-only solution fails to provide a valid PVT solution in case of jamMedium and jamStrong. 


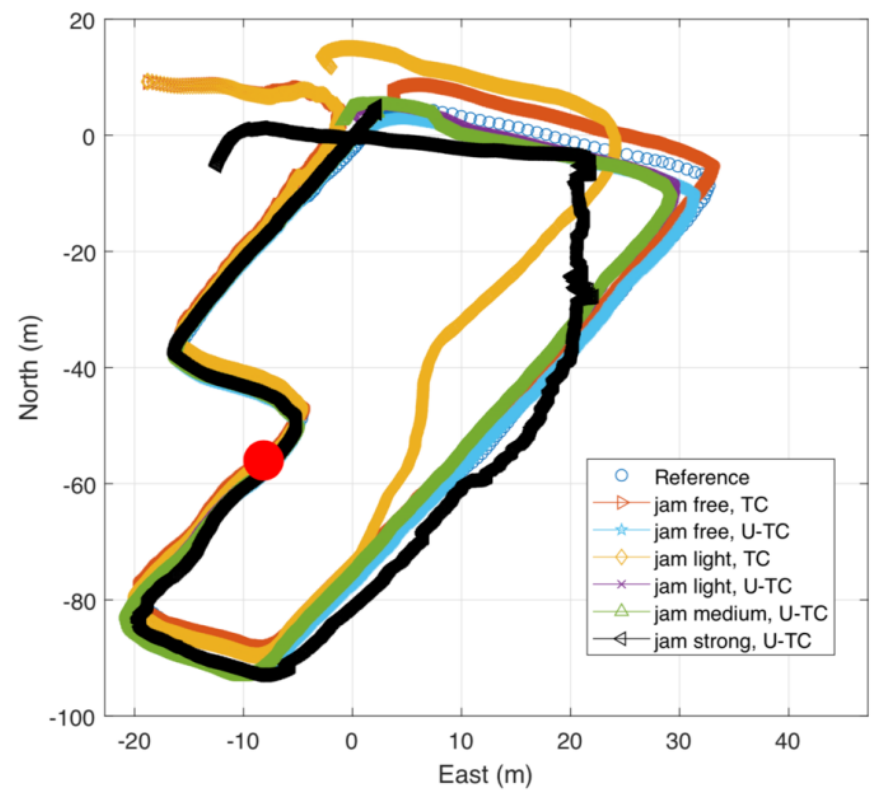

Figure 6: 2D trajectories for TC and U-TC integration algorithms. The red dot corresponds to the time when the jamming signals are injected.

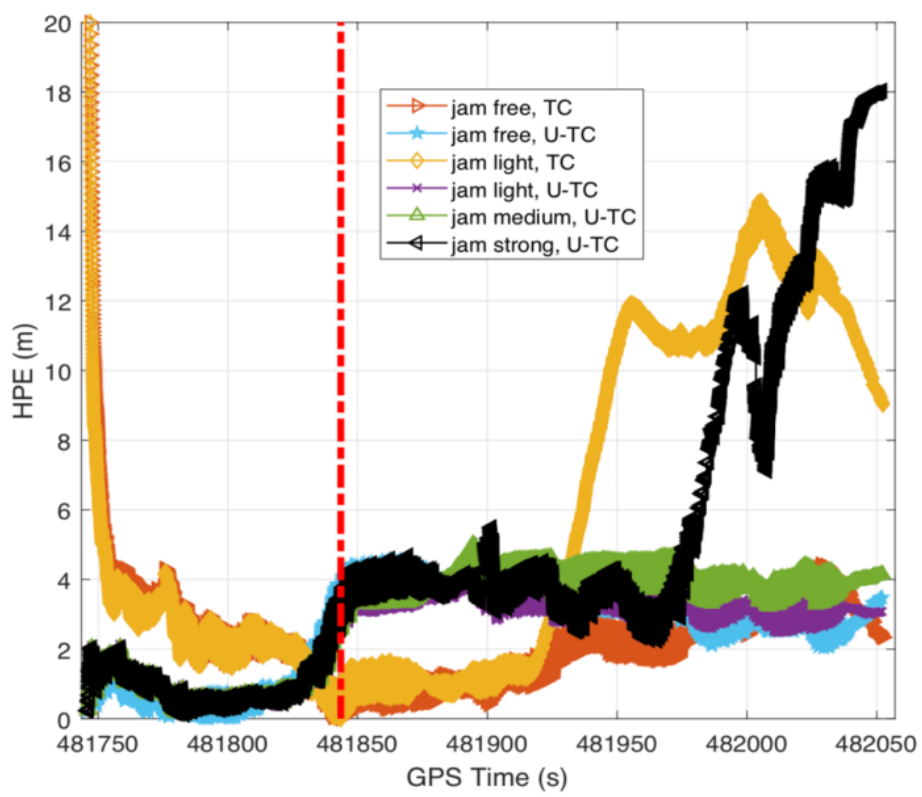

Figure 7: HPE over time for TC and U-TC integration algorithms. The red bar corresponds to the time when the jamming signals are injected.

In order to figure out and evaluate the benefits of having multi-sensors data, we have also run the software receiver without using the measurements coming from the camera and the odometer. In this case, both the TC and U-TC solution are obtained through GNSS and INS measurements only. With this receiver's configuration, the HPE results are summarized in Table 3. 
Table 3: Positioning performance assessment in case of GNSS/INS-only fusion

\begin{tabular}{|c|c|c|c|c|c|c|c|c|}
\hline & \multicolumn{2}{|c|}{ Jam free } & \multicolumn{2}{c|}{ Jam light } & \multicolumn{2}{c|}{ Jam medium } & \multicolumn{2}{c|}{ Jam strong } \\
\hline & TC & U-TC & TC & U-TC & TC & U-TC & TC & U-TC \\
\hline mean(HPE) (m) & 0.74 & 2.52 & 32.85 & 4.94 & - & 18.07 & - & - \\
\hline $\max ($ HPE) (m) & 1.96 & 3.91 & 80.81 & 9,47 & - & 34.40 & - & - \\
\hline $50^{\text {th }}$ percentile of HPE (m) & 0.67 & 2.69 & 40.81 & 4.92 & - & 18.01 & - & - \\
\hline $75^{\text {th }}$ percentile of HPE (m) & 0.91 & 2.96 & 57.22 & 7.44 & - & 29.32 & - & - \\
\hline $95^{\text {th }}$ percentile of HPE (m) & 1.39 & 3.77 & 74.71 & 9.17 & - & 33.52 & - & - \\
\hline Availability (\%) & 100 & 100 & 100 & 100 & 0 & 100 & 0 & 0 \\
\hline
\end{tabular}

By comparing the results shown in Table 2 and Table 3 and related to different hybrid receiver's configurations, it is evident the benefits of having a multi-sensor system, with respect to a GNSS/INS-only integration. The improvement in terms of performance, obtained through a multi-sensor receiver, gets more evident as the power strength of the jamming increases. In case of U-TC integration, the position performance is remarkably improved in case of both jamMedium and jamLight. In fact, the 95th percentile of the HPE is improved from $9.17 \mathrm{~m}$ (GNSS/INS) to $3.85 \mathrm{~m}$ (multi-sensor) for jamLight and from $33.52 \mathrm{~m}$ (GNSS/INS) to $4.55 \mathrm{~m}$ (multi-sensor) for jamMedium. Moreover, in case of jamStrong the U-TC GNSS/INS system is not able to provide a valid PVT while, adding more sensors, we are able to keep in-lock the tracking of the GNSS signals and to obtain a positioning solution.

The same can be said about the TC integration. In fact, the benefits of multi-sensor with respect to the GNSS/INS integration are consistent in case of jamLight. The $95^{\text {th }}$ percentile of the HPE, it is decreased from $74.71 \mathrm{~m}$ (GNSS/INS jamLight) to $13.76 \mathrm{~m}$ (multisensor jamLight). As expected, both the two configurations of the receiver fail to work in case of jamMedium and jamStrong

\section{CONCLUSIONS}

Aiming at taking advantage of the existing onboard technologies for ground vehicle navigation in challenging environments, this paper has developed an integrated camera/odometer/IMU/GNSS system based on the EKF design, according to an ultra-tightly coupled architecture.

Based on the EKF design, the proposed integrated system is able to provide 18-state navigation solutions. Our implementation has been validated through an outdoor test which provided raw GNSS data, visual measurements and IMU data as well as data from an odometer. In order to emulate jamming signals with different signal strengths, three different scenario datasets are created by using the Record and Replay approach, each characterized by different level of attenuation equal to $-30 \mathrm{~dB},-20 \mathrm{~dB}$ and $-10 \mathrm{~dB}$, and denoted respectively as jamLight, jamMedium and jamStrong. In scenarios affected by jamming interference, one possible countermeasure is offered by the intelligent integration of measurements from multiple sensors with complementary nature. Such hybrid positioning systems may be resilient to the jammer thus increasing the overall robustness. However, among the described integration strategies, the loose and tight coupling approaches do not provide a real mitigation solution against the jamming threat. In fact, it can prevent the GNSS receiver to provide observables' measurements and to compute the position fix. On the other hand, the ultra-tight coupling integration enables the tracking of signal with low $\mathrm{C} / \mathrm{N} 0$ enhancing the system's robustness to interference.

As far as the U-TC architecture is concerned, the camera/odometer/IMU/GNSS integration has outperformed the U-TC GNSS/INS scheme which has yielded no navigation solutions in case of jamStrong.

Due to the great number of sensors available nowadays (e.g. LiDAR, UWB, modern thermal cameras) they could be potentially exploited in the fusion algorithm as future work. Among the additional sources of information that can be fused, it is worth mentioning the recent results obtained by cooperative techniques that allows to have estimation of the baseline between vehicles, just using GNSS measurements. 


\section{REFERENCES}

1. Pullen, S., and Gao, G.X., "GNSS Jamming in the Name of Privacy, potential threat to GPS aviation," Inside GNSS, March/April 2012.

2. Groves, P.D., "Principles of GNSS, inertial, and multisensor integrated navigation systems," Artech house, 2013.

3. Hartley R., and Zisserman, A., "Multiple view geometry in computer vision," Cambridge university press, 2003.

4. Skog, I. and Handel, P., "In-car positioning and navigation technologies-a survey," IEEE Transactions on Intelligent Transportation Systems, vol. 10, no. 1, pp. 4-21, 2009.

5. Gao, Y., Liu, S., Atia, M., and Noureldin, A., "INS/GPS/LIDAR integrated navigation system for urban and indoor environments using hybrid scan matching algorithm," Sensors, vol. 15, no. 9, pp. 23286-23302, 2015.

6. Leppakoski, H., Collin, J., and Takala, J., "Pedestrian navigation based on inertial sensors, indoor map, and WLAN signals," Journal of Signal Processing Systems, vol. 71, no. 3, pp. 287-296, 2013.

7. MacGougan, G., O'Keefe, K., and Klukas, R., "Tightly-coupled GPS/UWB integration," The Journal of Navigation, vol. 63, no. 1, pp. 1-22, 2010.

8. Falco, G., Einicke, G.A., Malos, J.T., and Dovis, F., "Performance Analysis of Constrained Loosely Coupled GPS/INS Integration Solutions," Sensors 2012, Vol. 12, pp.15983-16007.

9. Falco, G., Pini, M., and Marucco, G., "Loose and Tight GNSS/INS Integrations: Comparison of Performance Assessed in Real Urban Scenarios," Sensors 2017, Vol. 17, pp.255-266.

10. Falco, G., Gutiérrez, C.C., Serna, E.P., Zacchello, F., AND Bories, S., "Low-cost Real-time Tightly-Coupled GNSS/INS Navigation System Based on Carrier-phase Double- differences for UAV Applications," Proceedings of the 27th International Technical Meeting of the Satellite Division of The Institute of Navigation (ION GNSS+ 2014), Tampa, FL, USA, 8-12 September 2014; pp. 841-857.

11. Gao, G. and Lachapelle, G., "A novel architecture for ultra-tight HSGPS-INS integration," Journal of Global Positioning Systems,vol. 7, pp. 46-61, 2008.

12. Jwo, D.J., Yang, C.F., Chuang, C.H. and Lin, K.C. "A Novel Design for the Ultra-Tightly Coupled GPS/INS Navigation System". Journal of Navigation 2012, Vol. 65, pp:717-747.

13. Wang, X., Li K., Gao, P., and Wang W., "Reinforced Ultra-Tightly Coupled GPS/INS System for Challenging Environment," Mathematical Problems in Engineering, vol. 2014, 12 pages, 2014.

14. Ruotsalainen, L., Kirkko-Jaakkola, M., Bhuiyan, Z., Soderholm, S., Thombre, S., and Kuusniemi, H., "Deeply coupled GNSS, INS and visual sensor integration for interference mitigation," Proceedings of the 27th International Technical Meeting of the Satellite Division of The Institute of Navigation (ION GNSS+ 2014), Tampa, FL, USA, 8-12 September 2014;, pp. 2243-2249.

15. Cristodaro, C., Ruotsalainen, L., and Dovis, F. "GNSS/INS/Visual Deeply Coupled Integration: Preliminary Investigation on Dynamic Jammed Datasets," Proceedings of the 31th International Technical Meeting of The Satellite Division of the Institute of Navigation (ION GNSS+ 2018). Miami, FL, 24-28 September2018, pp. 3088-3097.

16. Cristodaro, C., "Advanced Integration of GNSS and External Sensors for Autonomous Mobility Applications," PhD thesis, Politecnico di Torino, 2019.

17. Serant, D et al. "Field test performance assessment of GNSS/INS ultra-tight coupling scheme targeted to mass-market applications". Proceedings of the: 6th ESA Workshop on Satellite Navigation Technologies and European Workshop on GNSS Signals and Signal Processing, (NAVITEC), Noordwijk, Netherlands, 5-7 December 2012., pp. 1-8.

18. Jirawimut R. et al. "Visual odometer for pedestrian navigation," IEEE Transactions on Instrumentation and Measurement 52.4 (2003), pp. 1166-1173.

19. Ruotsalainen, L., "Vision-Aided Pedestrian Navigation for Challenging GNSS Environments," vol. 151. PhD thesis, Publications of the Finnish Geodetic Institute, Masala, Finland, 2013.

20. Cristodaro, C., Ruotsalainen, L., and Dovis, F., "Benefits and Limitations of the Record and Replay Approach for GNSS Receiver Performance Assessment in Harsh Scenarios," Sensors, 18(7), pp. 2189, 2018.

21. Cristodaro, C., Dovis, F., and Ruotsalainen, L., "The record and replay approach for GNSS receiver performance assessment in road environment," Proceedings of the 2017 International Technical Meeting of the Institute of Navigation, Monterey, CA, USA (pp. 1369-1375), 2017.

22. USRP B-210. Available online: https://www.ettus.com/product/details/ UB210-KIT (accessed on June 2019).

23. Ublox M8QCAM. Available online: https://www.u-blox.com/en/product/ evk-m8xcam (accessed on June 2019).

24. Xsense MTi-G-710. Available online: https://www.xsens.com/products/ mti-g-710/ (accessed on June 2019).

25. GoPro Hero5 Session. Available online: https:/it.shop.gopro.com/EMEA/cameras/ (accessed on June 2019). 